International Review of Community Development

Revue internationale d'action communautaire

\title{
Au-delà de la prévention : une pratique politique de rectification permanente
}

Association de recherche et de promotion en éducation populaire (ARPEP)

\section{Beyond prevention: a political praxis of continuing self-correction Research and Development Group for Popular Education.}

\section{Más allá de la prevención: Una práctica política de rectificación permanente \\ Asociación de investigación y de promoción en educación popular}

Bernard Francq et Françoise Goffinet

Numéro 11 (51), printemps 1984

La prévention, entre le contrôle et l'autogestion

URI : https://id.erudit.org/iderudit/1034636ar

DOI : https://doi.org/10.7202/1034636ar

Aller au sommaire du numéro

Éditeur(s)

Lien social et Politiques

ISSN

0707-9699 (imprimé)

2369-6400 (numérique)

Découvrir la revue

Citer ce document

Francq, B. \& Goffinet, F. (1984). Au-delà de la prévention : une pratique politique de rectification permanente : association de recherche et de promotion en éducation populaire (ARPEP). International Review of Community Development / Revue internationale d'action communautaire, (11), 169-173. https://doi.org/10.7202/1034636ar

\section{Résumé de l'article}

L'Association de recherche et de promotion en éducation populaire, située à Lille-Sud, travaille depuis 1974 dans un quartier à forte composante de population immigrée. Ayant eu une histoire singulière qui, de la mise en place d'un club de prévention à l'organisation de stages de préformation, a été marquée par des processus de rectification permanente, l'Association a développé des modes d'intervention centrés sur le développement des formes de solidarité active et d'auto-organisation des jeunes maghrébins.

Avec l'arrivée de la gauche au pouvoir, le travail de prévention basé sur la libre adhésion s'est trouvé dépassé par une politique de regroupement forcé, parfois concurrentiel, des partenaires. L'enjeu politique pour l'Association est constitué par la nécessité de réunir des partenaires en un réseau capable d'être porteur de projets globaux d'animation ou de production économique. 


\section{le socialisme et \\ la prévention : \\ l'expérience \\ française}

\section{Au-delà de la prévention : une pratique politique de rectification permanente (ARPEP)}

\section{B. Francq \\ F. Goffinet}

\section{Fiche de présentation}

Association de recherche et de promotion en éducation populaire (ARPEP)

Cadre juridique : Association loi 1901

Agréments :

Depuis juin 1977 : préfecture, travail, éducation nationale, formation permanente pour mener une action de préformation "bâtiment ", " mécanique ";

6 actions menées en 5 années; 90 adolescents touchés;
Depuis mars 1980 : la D.D.A.S.S. et la préfecture du Nord pour mener une action de prévention, quartier de Wazemmes;

Depuis janvier 1981 : agréments pour faire fonctionner deux ateliers de production-formation.

Interventions de I'ARPEP :

a) sur les groupes de population: par l'animation (mercredi enfants, carnaval, accueils et loisirs, camps, rattrapage scolaire, action femmes); b) coordination avec les autres travailleurs sociaux, municipalité, associations, organismes divers ;

c) actions de formation et de production pour jeunes adolescents :

1. préformation: stages d'accès à la vie professionnelle, travaux pratiques en mécanique, bâtiment, menuiserie, stages en entreprises ;

2. atelier de production : menuiserie, mécanique, tôlerie, automo- 
Q Votre association a une histoire singulière. Elle est depuis toujours un club de prévention faisant au départ un travail assez classique : insertion dans un quartier, accueil, activités d'animation diverses. Très rapidement, vous avez été amenés à participer activement à l'élaboration de politiques urbaines alternatives. Puis, dès 1977, vous vous êtes lancés dans les stages de préformation que le gouvernement de l'époque tentait timidement de mettre en place. Depuis l'arrivée de la gauche au pouvoir, vous organisez des stages d'insertion sociale et professionnelle aussi bien aux métiers du bâtiment qu'à ceux de l'animation et de l'éducation. À quoi correspond tout ce parcours? À une adaptation permanente aux politiques du pouvoir?

R Une chose est très importante pour nous : c'est que les processus de rectification sont permanents depuis la naissance du club; je crois qu'on peut les inscrire aussi dans I'histoire locale du mouvement ouvrier français depuis 1976 . On n'échappe pas à cette somme de contradictions qui ont été à l'oeuvre et qui ont fourni la possibilité pour d'anciens gauchistes de monter, à un moment donné, un club de prévention et de rectifier en permanence le type d'orientation politique qui avait été à l'oeuvre pendant plus de sept ans.

Q On pourrait voir votre parcours comme une insertion dans une politique d'hygiénisme psycho-médicosocial...

R Non. On n'a pas affaire à un prolongement d'hygiénisme psycho-médico-social mais à des processus de rectification politique par la transformation des situations politiques qui nous ont amenés à changer en permanence notre stratégie. On n'a pas voulu non plus se confiner dans une espèce d'idéalisme social du type " faire un petit Lip dans la région " ou monter le premier conseil ou soviet ouvrier! Au départ, notre axe de travail, à 
partir du club de prévention, a été l'auto-organisation des gens sur des bases revendicatives, au niveau de l'habitat, de l'école, des loisirs. Mais on a rencontré de nombreuses difficultés d'auto-organisation. Pour les expliquer, il faut tenir compte de plusieurs éléments qui tous renvoient au mouvement politique dans la société, auquel le mouvement associatif est étroitement lié. C'est ce qui peut expliquer nos processus de rectification permanente quant aux orientations de l'association.

Une première étape a été 1978 , moment où les deux grands partis ouvriers en France, le Parti communiste et le Parti socialiste, se sont mis d'accord sur un programme commun; pour une large fraction de la classe ouvrière et du réseau associatif, des changements sociaux importants devaient naître de cet accord. La stratégie était de dire : ce n'est pas la mobilisation à la base du secteur associatif qui va permettre de transformer ou de modifier le champ social, mais plutôt la politique, la pression du pouvoir politique qui va transformer de fait la société.

À la suite de l'échec du programme commun, l'ensemble du mouvement associatif et du mouvement ouvrier a vécu un repli important ; le mouvement associatif s'est adapté à la situation politique sur le territoire national. C'est un repli parce qu'en 1976, on pensait encore qu'il y avait possibilité d'auto-organisation des populations sous-prolétarisées. En 1978, une partie du réseau associatif croyait que le programme commun et la venue rapide des socialistes et des communistes au pouvoir allait permettre de résoudre toute une série de contradictions à l'oeuvre. Mais les choses ne se sont pas passées comme cela et l'ensemble du mouvement ouvrier et du mouvement associatif s'est retrouvé dans une situation de désarroi pendant un an ou deux, en même temps que se produisait une division syndicale et politique énorme que l'on a retrouvée au niveau du mouvement associatif.

Un deuxième élément explique la rectification de nos axes de travail : l'apparition de populations dont les formes d'auto-organisation ont été différées. Les gamins immigrés de $12-13$ ans par exemple, qu'on avait en 1976 et 1977, apparaissent aujourd'hui comme des leaders dans leur milieu; ce n'est pas qu'on soit de superéducateurs : on a fait un boulot normal dans un club de prévention un peu à part ; mais dans une situation politique " favorable ", ces jeunes peuvent commencer à s'auto-organiser. Ils sont sans doute la population la plus opprimée.

Les méthodes d'autoorganisation qu'on a essayé d'induire avec les jeunes du quartier, on les retrouve six ans après utilisées par les mêmes copains, capables aujourd'hui de monter leur propre association de jeunes immigrés à caractère politique très marqué. Quand on les a vus il y a six mois, au moment du lancement de leur association, on s'est rendu compte que tous les apprentissages (conduite de réunion, prise de parole, etc.) s'étaient faits à cette époque-là.

Il faut tenir compte d'un troisième élément : l'approfondissement de la crise économique. Les gens qu'on touche aujourd'hui ne sont plus du tout les mêmes que ceux qu'on a touchés en 1976 ou 1977. Les phénomènes de crise s'accompagnent de phénomènes de paupérisation; les gens qui n'étaient pas déclassés en 1976 le sont en 1984. Pour nous, tout cela $a$ induit des processus de rectification qui ne sont pas là simplement pour expliquer l'évolution d'un club de prévention. Chaque fois, il y a des processus de rectification permanente où plusieurs facteurs se combinent. C'est la nature d'une structure associative comme la nôtre d'être capable à un moment donné de répondre aux problèmes nouveaux qui se posent. Là où on peut dire que globalement on a réussi en permanence à rectifier et à réorienter la politique de l'association, c'est que de 6 salariés, on est passé à 21. C'est un changement quantitatif qui est significatif de cette adaptation.

Q Le travail de prévention mené par des clubs ou des associations volontaires a-t-il connu l'évolution que vous avez choisie?

R Au départ, l'idéologie du travail de prévention, c'était avant tout un travail de relation, un travail de contact mené sur un milieu dit " malade ", délinquant, etc., qui ne se positionnait pas en fonction de situations politiques. Les sociologues se sont très vite interrogés sur la nature d'une action repliée sur elle-même qui étiquetait des populations par un vocabulaire thérapeutique, avec ses principes de libre adhésion, d'écoute... A ce propos, il n'y a jamais eu de "psy " dans notre équipe. Maintenant, on peut dire que la prévention organisée en clubs subsidiés fait partie du discours dominant. Encore une fois, c'est par rapport au processus de rectification de l'intervention sur un milieu que les enjeux se sont définis. Certains clubs ont effectué cette rectification, d'autres se sont développés à partir du discours dominant de l'intervention sociale; ils ont eu des attitudes opportunistes à chaque virage de la logique politique - étatique - d'intervention sociale. II y a six mois, par rapport au conseil communal de prévention, nous disions : "Ce n'est pas forcément mauvais, tout dépend comment on peut organiser le réseau pour ne pas contrecarrer le pouvoir des gens dans les quartiers. " Eux disaient : " Non! déontologie oblige,libre adhésion, c'est hors de question. " Aujourd'hui ils changent d'avis pour des raisons qui ne sont ni d'ordre déontologique ni d'ordre pédagogique : ils savent que les modes de finance- 
ment des clubs de prévention vont changer, et que s'ils ne sont pas capables de s'allier objectivement et ponctuellement au pouvoir politique - le financement va venir du conseil général - il vont se faire avoir. Ce qui veut dire que dans le Nord, il n'y a jamais eu de définition d'une politique de réseau, capable d'enfoncer le même clou au même moment par rapport aux pouvoirs politiques ou financiers. C'est plutôt un système de concurrence permanente pour la préservation d'une structure existante en matière de prévention qui a régné.

Q Outre ces processus de rectification permanente, qu'est-ce qui caractérise votre mode d'intervention?

R Les gens n'ont pas dans leur tête une notion de prévention qui consisterait à se demander : de quoi va-t-on nous prévenir? II y a plutôt des liens d'intérêts objectifs entre les gens du quartier et nous. Le club de prévention est devenu - et c'est là qu'il s'est différencié fondamentalement des autres - non pas un lieu thérapeutique, mais un lieuressource, où on peut trouver des solutions à des situations d'urgence de type économique ou des solutions en termes de formation.

Pour la population, pour les gamins auxquels on s'adresse, on a réussi à créer toute une série de liens d'intérêts avec le quartier et l'environnement. Par le moyen des stages de préformation, des ateliers, des services rendus (les sorties...), l'association apparaît pour le quartier comme recours possible au plan économique et humain.

Notre mode d'intervention a deux particularités : il essaie de développer en permanence des formes de solidarité actives avec le public, par des liens de l'ensemble de l'équipe avec l'ensemble des organisations ouvrières. En outre, il met l'association à disposition comme un groupe-ressource où les gens peuvent trouver des réponses concrètes à leurs problèmes. Q Votre public, ce sont principalement des jeunes maghrébins peu scolarisés. Quel problème rencontrez-vous dans l'organisation de vos actions de formation?

R Le problème pour nous, c'est notre capacité de multiplier des formes de services et de trouver de nouvelles ressources. On a mis sur pied une filière qu'on a fait valider par un diplôme. Notre petite association a pris contact avec l'Éducation nationale - ca a duré six mois - et elle est arrivée à obtenir des C.A.P. (Certificat d'aptitude professionnelle) par unités capitalisables. Étant donné le bas niveau de formation des jeunes, on s'est dit qu'ils auraient d'énormes problèmes pour accéder à la qualification. On a alors pensé recourir à des modes de support informatique d'enseignement assisté par ordinateur qui s'intègrent dans nos actions de formation. II n'est pas question de nous situer en concurrence par rapport à l'Éducation nationale! Ce qui nous intéresse, c'est de pouvoir nous associer, dans un processus ou un dispositif de formation, avec l'Éducation nationale car si on veut avoir une logique pédagogique, il faut assurer une filière de formation et qualifier les jeunes, sans quoi ce n'est pas la peine de faire de la formation. Du relationnel on peut en faire sans faire de formation! Mais une fois que le problème de la formation est posé, il faut obliger toute une série de partenaires à intervenir.

Q Par rapport à la crise, les formations que vous mettez en place ne contribuent-elles pas à marginaliser davantage les jeunes immigrés qui sont votre public principal? $\mathbf{R}$ Je crois qu'on y contribuerait si on avait un type d'intervention médical ou de nature psychiatrique. En fait, la recherche de solutions en termes économiques permet d'aller plus loin. Avec un public très marginalisé, exclu, qui a des difficultés énormes de communication avec l'environnement, on peut sortir d'une problématique de renforcement de la marginalisation, on arrive à augmenter sa capacité sociale à identifier ce qu'il doit connaître pour travailler normalement, à ne pas lui apprendre à utiliser les filières de dépendance qu'organisent systématiquement les structures de chômage. Le minimum d'augmentation de capacité sociale, de qualification sociale qu'on parvient à produire chez les gens sur des dimensions, non pas thérapeutiques ou psychiatriques, mais de confrontation et d'adaptation au réel, crée des avancées intéressantes.

Quelque part, ça a permis de donner corps à une forme d'autoorganisation et d'intervention politique de ces jeunes Arabes sur leur environnement. Plutôt que de marginaliser, je crois qu'on augmente la qualification stratégique des jeunes. Aujourd'hui il est très difficile de théoriser ce qu'est une action de prévention : elle doit être liée en permanence à l'environnement ; elle doit aussi s'interroger sur les services ou les ressources mises à disposition et sur la capacité de rectification permanente du dispositif par rapport à l'apparition de nouvelles conditions économiques ou politiques.

Q Avec l'arrivée de la gauche au pouvoir, avec le renforcement d'une politique de formation pour les jeunes " marginalisés ", qu'est-ce qui a changé réellement?

R Avec la présence du P.S. au 
pouvoir, le contexte politique est plus favorable que si la droite était au pouvoir. Mais le gouvernement doit à la fois prendre des mesures d'austérité et développer des formes de solidarité actives et permanentes avec le réseau associatif, s'il veut conserver un minimum de crédibilité. II débloque effectivement toute une série de crédits pour le réseau d'intervention sociale. Et il oblige - c'est là la contradiction toute une série de partenaires à se coordonner politiquement pour pouvoir bénéficier de ces crédits. L'exemple type, ce sont les opérations Été 82 et 83 . Le gouvernement ne négocie même plus des financements avec une association, il les négocie avec un collectif dans un quartier sur la base d'un projet de loisirs commun pour juillet et août. Il crée lui-même des formes de solidarité actives qui vont entrer, à un moment ou à un autre, en concurrence avec sa politique d'austérité économique.

Notre projet consiste aujourd'hui à utiliser cette capacité-là pour pouvoir générer une première tentative d'organisation du réseau sur la ville de Lille. Mais, c'est très difficile.

Par exemple, une association avait un projet d'aménagement urbain ; une autre avait un projet de construction d'habitats modulaires; de notre côté nous avions un stage bâtiment et un stage de métiers d'animation/éducation. Nos projets ne sont pas concurrentiels. On s'est donc regroupés pour obliger le politique à financer ces opérations. II n'a plus affaire à un seul partenaire, mais à une association qui a un projet global d'animation, de formation et de production.

Cependant, par rapport au politique, on est coincé ! On a toujours un pas d'avance par rapport à d'autres, mais les élus locaux ne sont pas idiots, ils veulent aller vers des solutions économiques. On est intéressant pour le P.S. parce qu'effectivement, on a des choses à montrer.
Q Ça veut dire aussi que vous pouvez servir de laboratoire par rapport aux solutions à apporter à la crise économique?

R La crise a fait déraper l'école, les formations ; elle a créé des problèmes impossibles dans les lieux d'habitat. En fait, elle a tout fait éclater. On s'en accommode, on en vit quelque part. On était un peu en avance par rapport à l'idéologie dominante, maintenant on est en plein dedans. L'idéologie de la prévention telle que définie il y a dix ans n'est plus celle d'aujourd'hui. On a servi de laboratoire d'expérimentation avec l'arrivée de la gauche au pouvoir. Le pouvoir, les élus locaux nous citent en exemple en disant : voilà ce que devrait être un travail de prévention; montez des stages de formation, faites.des qualifications, montez des ateliers, faites de l'informatique, etc. D'un autre côté, ça nous sert contradictoirement à pouvoir négocier avec les élus politiques de façon stratégique, puisqu'on peut être au centre de tout un regroupement de partenaires sur des projets de type économique ou de type animation globale. On est à la charnière de ce qui se fait. On est lié à toute une série de projets importants et fondamentaux pour la politique, qui reposent c'est là que ça devient une contradiction énorme pour nous - simplement sur notre capacité d'action et sur la logistique qu'on a mise en place depuis des années. Ça va même plus loin que ça, puisque les élus politiques nous demandent de détacher une partie de notre personnel et de le mettre à la disposition des missions locales pour l'emploi afin de mettre en place leur propre projet politique, alors qu'ils savent pertinemment où nous nous situons politiquement. Toute notre difficulté va être d'utiliser cette place stratégique pendant un moment pour réaliser effectivement un réseau constitué capable d'être porteur de projets d'animation ou de production.
On veut favoriser l'émergence d'un réseau associatif dans le champ politique qui nous est laissé, très étroit de toute façon. II sera en avance de deux ou trois ans sur l'arrivée de la droite au pouvoir! La gauche a un gros problème : c'est qu'elle ne trouve aucun relais capable de faire des actions concrètes sur le terrain... ce n'est pas un élu municipal qui est capable avec deux ou quatre gamins de construire un hangar! Ce ne sont pas non plus les associations vieillotes qui vont faire ça, ni les travailleurs sociaux qui sont dépassés par les faits. L'enjeu, c'est que depuis deux ans, une politique de regroupement forcé, parfois concurrentiel mais déterminant des capacités de financement, crée des possibilités réelles d'association, de réseau entre des partenaires qui n'ont plus rien à voir avec la prévention ou avec les professionnels de celle-ci.

Pour nous, il y a là un problème politique énorme : on ne peut pas continuer à être un petit îlot sur la ville de Lille où on porte des projets à 80 heures par semaine. II faut coordonner le secteur associatif qui bouge, qui veut monter des projets, et amener les financiers et les politiques à soutenir ces projets-là. C'est très clairement pour nous un enjeu politique qui se situe bien audelà de la prévention.

(Propos recueillis par Bernard Francq et Françoise Goffinet) 